\title{
Automated Glaucoma Screening in Retinal Fundus Images
}

\author{
Srinivasan Aruchamy, Partha Bhattacharjee and Goutam Sanyal \\ CSIR-CMERI, National Institute of Technology \\ srinivaspsg@gmail.com,partha11cmeri@gmail.com,nitgsanyal@gmail.com,
}

\begin{abstract}
Glaucoma is an eye disease which damages the optic nerve that carries information from the eye to the brain. Glaucoma is the second foremost reason of blindness. An efficient hardware based implementation of glaucoma screening is a significant task in the automated retinal image analysis method. This paper presents an automatic glaucoma screening using a TMS320C6416DSK DSP board. The detection procedure consists of two stages. The first stage comprises of image pre-processing and detection of optic nerve head center using circular Hough Transform .In the second stage, the optic disk diameter is calculated and cup is segmented from disk. The proposition between disk and cup is calculated for abnormal image screening. The implemented technique is tested on a publicly available retinal image data sets and the average accuracy achieved is $97.5 \%$.
\end{abstract}

Keywords: fundus image, glaucoma, optic disk, circular hough transform, TMS320c6416

\section{Introduction}

Glaucoma is an irreversible eye syndrome. According to reports in 2010, it is second primary reason of blindness in the world. $2.3 \%$ of the peoples got affected from total population. It is predicted that this number will increase to $2.86 \%$ in 2020 . Studies have been shown that increase in intraocular pressure (IOP) of the eye is one the cause for glaucoma [2]. To maintain healthy vision, eye produces a small amount fluid called aqueous humor the same amount fluid will be thrown out of eye. This balance keeps the IOP in limit. If the balance is not maintained the IOP increase and damage the optic nerve head which make irreversible vision loss. So, the early precise detection and treatment of glaucoma will control the progression of the disease. The ophthalmologist uses the ratio between optic cup and optic disk (CDR) as a parameter to screen the glaucoma patients. The CDR is $<0.65$ in case of normal eye and for the glaucoma eye the value is lies in between 0.65 to 0.9. Manual glaucoma screening is time consuming and prone to human error. So, An intricate algorithm is needed for mass screening of glaucoma.Figure 1 shows the normal image and glaucoma image.
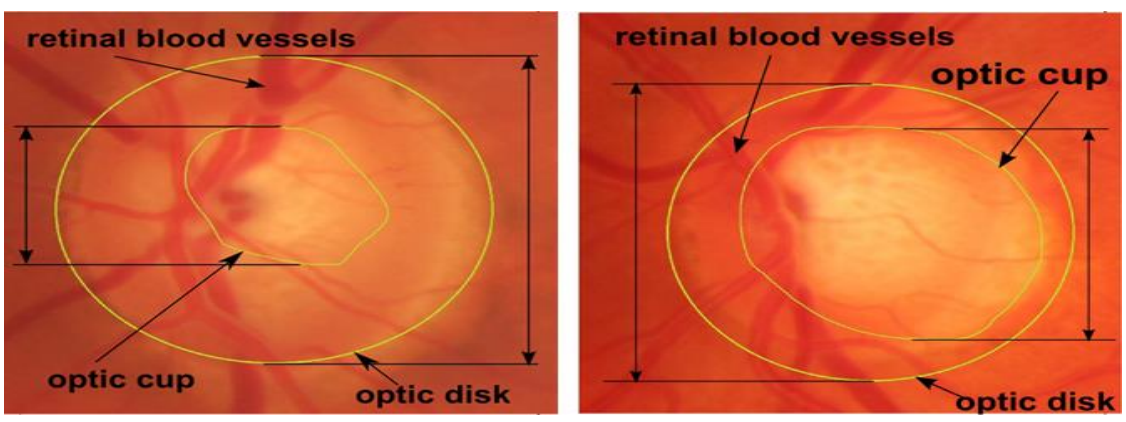

Figure 1. Retinal Image Marked with Optic Disk and Optic Cup 
Xiaoyang Song et al [3] developed an rule based model using fuzzy rules for large scale screening of glaucoma, where optic nerve defect detection and visual field examination are done separately to study sensitivity of the developed system. Jun Cheng et al [4] used machine learning method to screen glaucoma by calculating focal angle and focal length between iris and cornea. Dnyaneshwari D.P et al [5] used k-means clustering technique for detection of glaucoma images. The clustering algorithm used to find the area of optic of cup region as well as disk, and the proposed method achieved 97\% accuracy in glaucoma screening. Nilanjan dey et al [6] proposed harris corner method to find CDR and they achieved the accuracy of 95\%.Gayathri proposed ANN based classifier for glaucoma image classification, where structural and energy features were input parameters to the classifier and their method achieved the accuracy of $97.6 \%$ [7]. Tehmina Khalil et al [8] did a detailed study on glaucoma image processing in different level. The survey includes feature extraction, selection and machine intelligence techniques. Their method achieved $85 \%$ accuracy. Siamak Yousefi et al [9] used longitudinal series of structural data from patient eye as input to different machine learning methods, like Bayesian, Lazy, Meta and Tree. The combination of pixel arrangements and practical features were chosen by the classifiers and ranking has been done accordingly, which are deeply analyzed to classify glaucoma. Azin Poshtyar was proposed a method were the optic disk is segmented using template matching and appearance-based techniques. From the segmented optic disk, the cup is extracted. The cup to disk ratio is calculated for screening glaucoma images [10]. Most of the proposed techniques are implemented using simulation based software's. So, an attempt is made in our work to implement the algorithm in both hardware based platform and simulation software and the performance of the proposed method is analyzed.

\section{Proposed Method}

Retinal images are collected from the standard retinal database [11]. The RGB images of retina are taken as an input image, and then it was converted to gray scale image. From gray scale image the green channel image is taken for further processing. The image is converted to a header file format then loaded in to SDRAM of the DSP board. Image enhancement is performed by CLAHE block [12]. To reduce the computation complexity the optic disk is localized by cutting out several parts of the image, which are likely to be far from the OD. From the localized optic disk the edges were identified using canny edge detection technique. Circular Hough transform is performed on edge image to find the center of the optic disk and optic cup form the localized image. The circle (OD) is superimposed on the original image to mark the optic disk and cup. The cup to disk ratio is calculated and analyzed for screening glaucoma. The overall method is depicted in Figure 2.

\section{Pre-Processing and Optic Disk Segmentation}

Retinal images captured from fluorescence ophthalmoscope are of low gray level contrast. So, it is necessary to improve the image feature to make the subsequent image analysis easier. CLAHE [12] is a regular pre-processing scheme for image analysis, as it is extremely successful in building the region of interest more evident. 


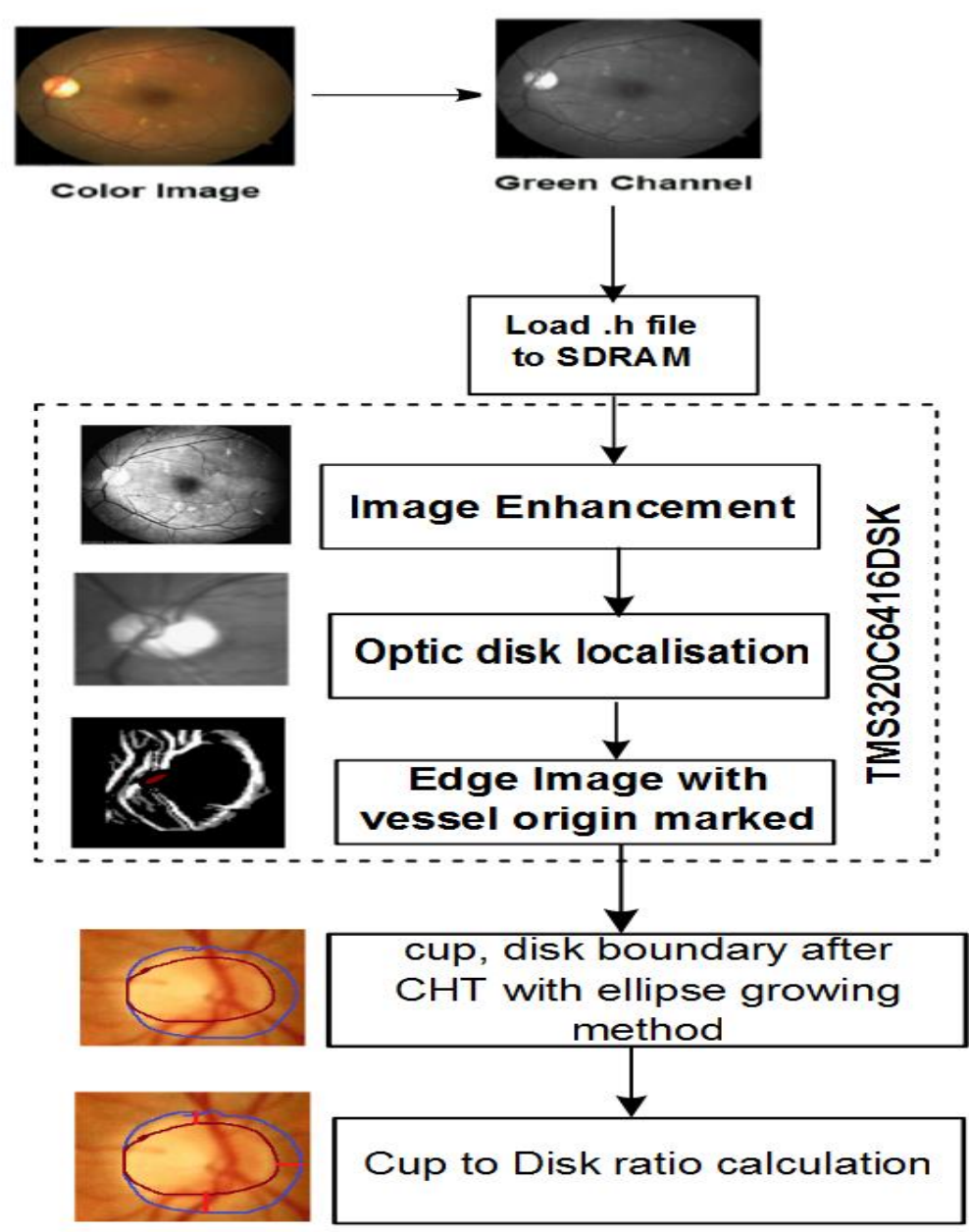

Figure 2. Schematic Diagram of Proposed Method

The retinal fundus image is divided into sub-images based on window size (n). The window size $\mathrm{n}$ is determined based on the understanding of the Optic disk volume and maximum diameter of the Optic disk in a database. As the intensity values of the pixels in the neighborhood of Optic disk is very high compared to other areas, the image is divided into $\mathrm{n}$ uniform windows and then the mean of each window is computed using equation 1 .

$$
m(i)=1 / N \sum_{k=0}^{N} I(k)
$$

Where $\mathrm{m}$ (i) is mean of $\mathrm{i}^{\text {th }}$ window, $\mathrm{N}$ is the total number of pixels in $\mathrm{i}^{\text {th }}$ window. Then the optic disc is located based on the highest mean. But when number of exudates is more (in case of DR) one window may not be able to locate optic disc properly. Also one window of prescribed size may not cover the whole optic disk. Through repeated experimentations it has that the window size needs to be at least $3 / 4^{\text {th }}$ of the optic disk for detecting and locating the same. The Figure 4 shows the localized optic disk from the green channel retina image. After the localization the edges were extracted using canny edge detector [13]. This edge detection process computes the gradient of image $f(x, y)$ by convolving it with the first derivative of Gaussian in $x$ and a $y$ direction to smooth noise and then computes the edge strength and direction for each pixel in the localized image. Figure 3(a), 3(b) shows the canny edge detected image from localized image.

$$
f_{x}(x, y)=f(x, y) *\left(\frac{-x}{\sigma^{2}}\right) e^{\frac{-\left(x^{2}+y^{2}\right)}{2 \sigma^{2}}}
$$


Where $\sigma=1.4$

$$
f_{y}(x, y)=f(x, y) *\left(\frac{-y}{\sigma^{2}}\right) e^{\frac{-\left(x^{2}+y^{2}\right)}{2 \sigma^{2}}}
$$

$$
\begin{aligned}
G & =\sqrt{f_{x}(x, y)^{2}+f_{y}(x, y)^{2}} \quad(\text { Edge strength) } \\
\theta & =\arctan \left(f_{y}(x, y) / f_{x}(x, y)\right)(\text { Edge direction) }
\end{aligned}
$$

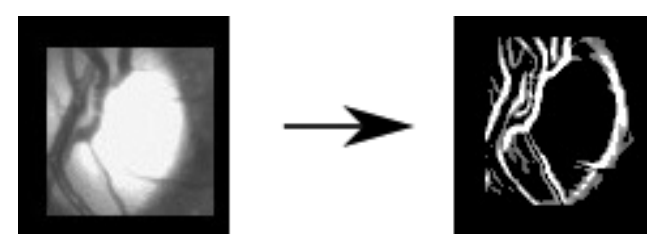

Figure 3. (a) Localized Image 3(b) Canny Edge Detected Image

The partial derivative $f_{x}, f_{y}$ is approximated using sobel operator of size $5 \times 5$. The Figure 4(a) shows the input image and Figure 4(b) shows the sobel filtering image.
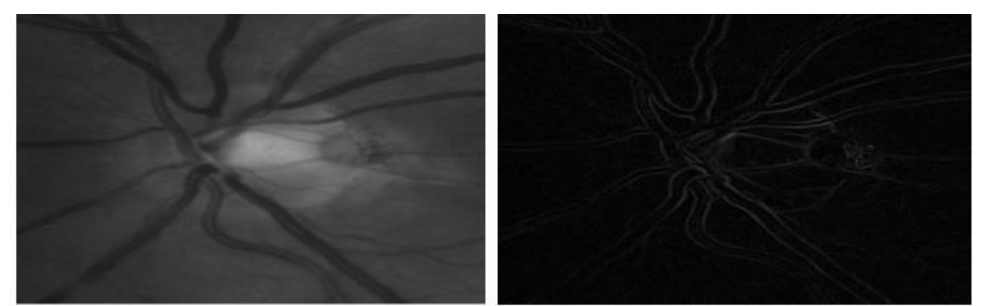

Figure 4. (a) Input Image 4 (b) Strength of Gradient Image by Sobel Operator

\section{Circular Hough Transform (CHT)}

Duda et al [14] proposed CHT, It is a modified version of Hough Transform (HT). Circle shape of the optic disk is computed using circle equation.

$$
r^{2}=(x-a)^{2}+(y-b)^{2}
$$

Where $r$ represents the radius of the circle and $(a, b)$ represents coordinates, which is the center of the circular object. To find out a circular disk in the image it is required to collect votes in three dimensional space $(a, b, r)$. CHT transforms the CHT transforms the image coordinate parameters into set of collected votes in the constraint space. Followed by every dot in the votes are calculated and accumulated in the group for all combination. Highest voting point will be considered the center of the circle and the coordinate points are show in the equation (6), (7) and (8). Figure 5 shows the example of conventional CHT.

$$
\begin{aligned}
& x=a+r * \cos (\theta) \\
& y=b+r * \sin (\theta)
\end{aligned}
$$

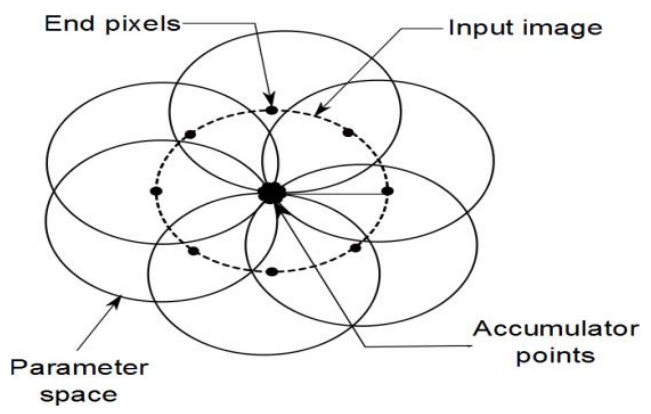

Figure 5. CHT with End Pixels and Accumulator Points 


\section{Optic Cup to Disk Ratio Calculation (CDR)}

The area of optic disk and optic cup are primary values in CDR [15] calculation. The optic disk is marked by using CHT. CHT provides the radius $r$ after circle detection. CDR is calculated using the equation 9 . The optic disk height is nothing but the diameter.

$$
\text { Cup to Disk Ratio }(C D R)=\frac{\text { optic cup height }}{\text { optic disk height }}
$$

Figure 6(a) to 6(c) shows the segmented optic disk from original image. The cup region will be increased whenever there is progression of glaucoma.

From the segmented optic disk boundary a $17 * 17$ pixels mask is created to approximately localize cup. The size of mask was determined empirically by calculation the area of the cup for 380 retinal fundus images.
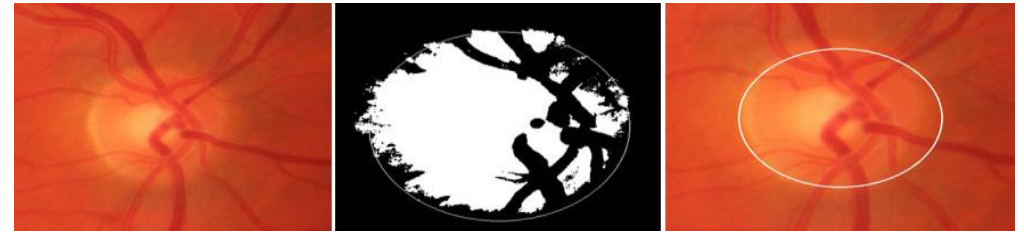

\section{Figure 6. (a) Original Image (b) CHT Image (c) CHT Super Imposed with Original Image}

\section{Experimental Setup and Implementation}

The proposed method was implemented in TMS320C6416 DSP board to ensure fast execution. So, that it will be helpful for mass screening of glaucoma.TMS320C6416DSK DSP [16], having following features, clock rates of up to $1 \mathrm{GHz}, 16 \mathrm{MB}$ of SDRAM and $512 \mathrm{~KB}$ of NV Flash Memory with JTAG interface for host communication. Code composer studio is used as host interface to exchange control information and data. The complete experimental setup is shown in the Figure 7.

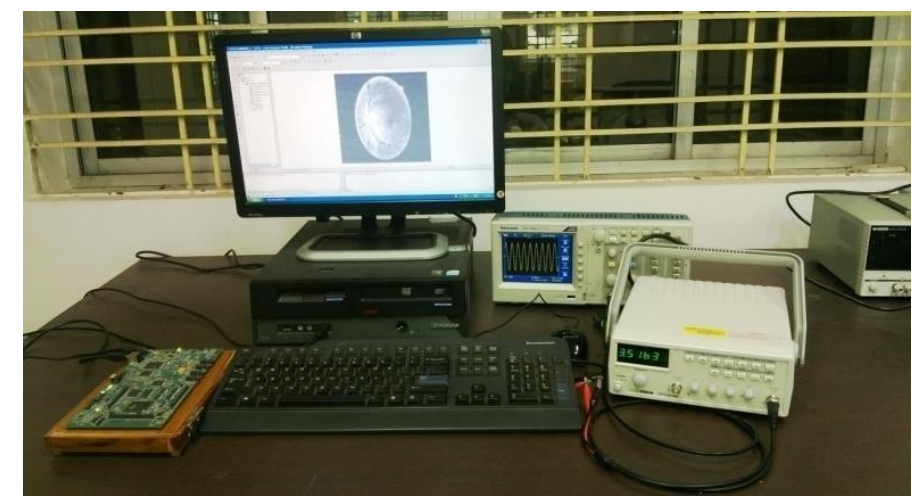

Figure 7. Hardware Setup

\section{Results}

The retinal images were taken from publically available database [11] which consists of 110 images. These images are having resolution of 600x400 pixels captured using colour retinal fundus camera. Ophthalmologists marked the Optic disks in these images and those were taken to train the algorithm. The Figure 8 shows the series of sample outputs from the proposed glaucoma screening method. 


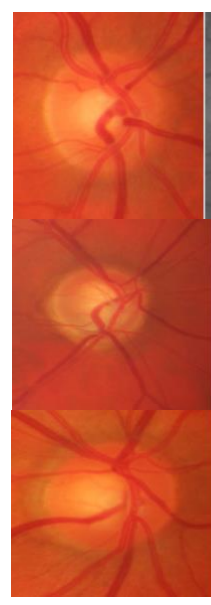

(a)

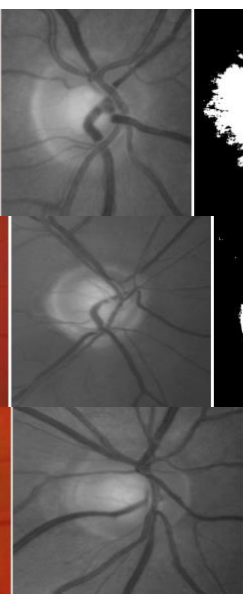

(b)

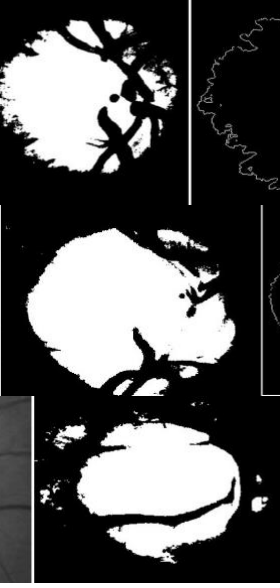

(c)

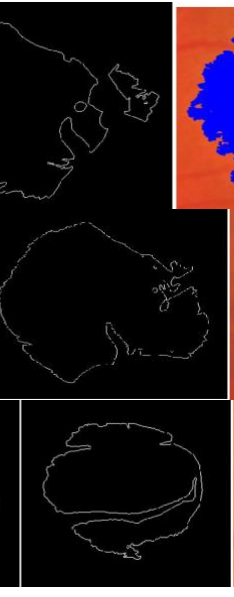

(d)

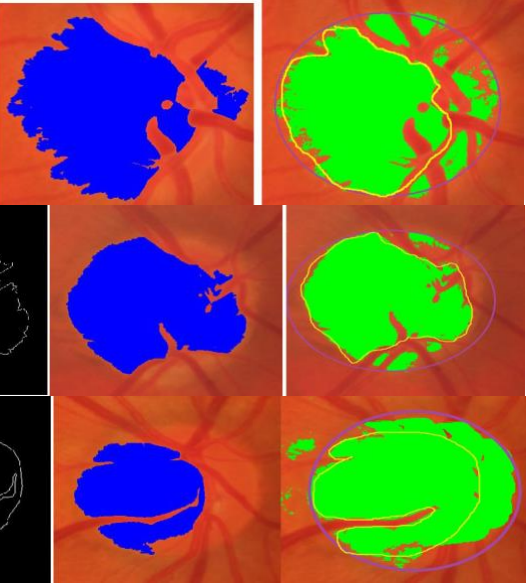

(e)

(f)

Figure 8. Sample Results from Left to Right: (a) Original Image, (b) Green Channel Image, (c) Optic Disk Segmented, (d) Optic Cup Edge Image, (e) Super-Imposed Image of Cup, (f) Optic Cup and Disk Marked Image

From the database 15 image simples results were shown in the Table 1 which contains the height of optic disk, optic cup and CDR values. According to our experiments it is seen that the CDR value is calculated and checked, If CDR $<0.65$ then those images are grouped into normal images. The values lies between 0.65 to 0.9 are grouped as glaucoma images. In our experiment total 110 images were tested and achieved the accuracy of $97.5 \%$.

Table 1. Cup to Disk Value of the Proposed Method

\begin{tabular}{|c|c|c|c|c|c|c|}
\hline \multirow{2}{*}{ S1.No } & \multicolumn{3}{|c|}{ Normal Eye } & \multicolumn{3}{c|}{ Glaucoma Eye } \\
\cline { 2 - 7 } & $\begin{array}{c}\text { Optic Disk } \\
\text { (height) }\end{array}$ & $\begin{array}{c}\text { Optic Cup } \\
\text { (height) }\end{array}$ & CDR & $\begin{array}{c}\text { Optic Disk } \\
\text { (height) }\end{array}$ & $\begin{array}{c}\text { Optic Cup } \\
\text { (height) }\end{array}$ & CDR \\
\hline 1 & 193 & 87 & 0.4519 & 201 & 133 & 0.6617 \\
\hline 2 & 183 & 101 & 0.5534 & 185 & 139 & 0.7534 \\
\hline 3 & 192 & 119 & 0.6198 & 184 & 131 & 0.7120 \\
\hline 4 & 168 & 102 & 0.6090 & 166 & 117 & 0.7069 \\
\hline 5 & 186 & 109 & 0.5860 & 204 & 141 & 0.6912 \\
\hline 6 & 208 & 95 & 0.4567 & 182 & 132 & 0.7253 \\
\hline 7 & 188 & 94 & 0.5000 & 199 & 131 & 0.6583 \\
\hline 8 & 215 & 101 & 0.4698 & 179 & 144 & 0.8045 \\
\hline 9 & 212 & 123 & 0.5802 & 171 & 116 & 0.6804 \\
\hline 10 & 200 & 109 & 0.5450 & 192 & 127 & 0.6615 \\
\hline 11 & 197 & 121 & 0.6158 & 180 & 136 & 0.7556 \\
\hline 12 & 207 & 111 & 0.5362 & 204 & 136 & 0.6667 \\
\hline 13 & 184 & 119 & 0.6467 & 193 & 130 & 0.6753 \\
\hline 14 & 187 & 78 & 0.4171 & 162 & 143 & 0.8827 \\
\hline 15 & 213 & 132 & 0.6197 & 190 & 125 & 0.6596 \\
\hline
\end{tabular}

\section{Conculsion}

A Novel method for glaucoma screening is presented and implemented using a dedicated hardware platform which is highly helpful to an ophthalmologist. Usage of the special hardware (TMS320c6416) also speeds up the detection process to a large extent. Proposed method achieved considerable improvement in accuracy in finding glaucoma images from the pool of retinal images and greater improvement in the screening process. 
The future scope of this work is interfacing the retinal image acquisition system into the DSP processor, which will provide real-time results of the glaucoma screening.

\section{Acknowledgements}

This research work was part of CSIR twelfth five year plan project (ESC0203/03). The authors are highly grateful for CSIR for their financial grand. The authors are highly grateful to Dr. Debdulal Chakraborty, Consultant (Incharge Vitreo Retina Services), Disha Eye Hospital Kolkata for his professional help to this research work.

\section{References}

[1] H. A. Quigley and A. T. Broman, "The number of people with glaucoma worldwide in 2010 and 2020", British Journal of Ophthalmology, vol. 90, no. 3, (2006), pp. 262-267.

[2] J. Kanski, B. Bowling, K. Nischal and A. Pearson, "Clinical Ophthalmology: A Systematic Approach: 7th Edition", Elsevier, (2011).

[3] X. Song, K. Song and Y. Chen, "A Computer-based Diagnosis System for Early Glaucoma Screening", Proceeding of the 2005 IEEE Engineering in Medicine and Biology, Shanghai, China (2005).

[4] J. Cheng, J. Liu, D. W. Kee Wong, N. M. Tan, B. H. Lee, C. Cheung, M. Baskaran, T. Y. Wong and T. Aung, "Focal Edge Association to Glaucoma Diagnosis", 33rd Annual International Conference of the IEEE EMBS, Boston, Massachusetts USA, August 30 - September 3, (2011).

[5] D. D. Patil, Dr. R. R. Manza, G. C. Bedke and D. D. Rathod, "Development of Primary Glaucoma classification technique using optic cup \& disc ratio", International Conference on Pervasive Computing (ICPC), (2015).

[6] N. Dey, A. B. Roy, A. Das and S. S. Chaudhuri, "Optical Cup to Disc Ratio Measurement for Glaucoma Diagnosis Using Harris Corner", Third International Conference on Computing, Communication and Networking Technologies (ICCCNT'12) 26th_28th, Coimbatore, India, July (2012).

[7] Gayathri R., P. Y. Rao and Aruna S., "Automated Glaucoma Detection System based on Wavelet Energy features and ANN, International Conference on Advances in Computing", Communications and Informatics (ICACCI), (2014).

[8] T. Khalil, S. Khalid and A. M. Syed, "Review of Machine Learning Techniques for Glaucoma Detection and Prediction", Science and Information Conference 2014, London, UK, August 27-29, (2014).

[9] S. Yousefi, Member, IEEE, M. H. Goldbaum, M. Balasubramanian, T. P. Jung, R. N. Weinreb, F. A. Medeiros, L. M. Zangwill, J. M. Liebmann, C. A. Girkin, and C. Bowd, "Glaucoma Progression Detection Using Structural Retinal Nerve Fiber Layer Measurements and Functional Visual Field Points", IEEE Transactions on Biomedical Engineering, vol. 61, no. 4, April (2014).

[10] A. Poshtyar, H. Ahmadieh and J. Shanbehzadeh, "Automatic Measurement of Cup to Disc Ratio for Diagnosis of Glaucoma on Retinal Fundus Images", 6th International Conference on Biomedical Engineering and Informatics, BMEI (2013).

[11] http://www.ia.uned.es/ ejcarmona/DRIONS-DB.html.

[12] K. Zuiderveld, "Contrast limited adaptive histogram equalization”, Graphics gems, J. G., vol. 4, (1994), pp. 474-485.

[13] J. Canny, "A computational approach to edge detection. Pattern Analysis and Machine Intelligence", IEEE Transactions on, PAMI, vol. 8, no. 6, November (1986), pp. 679-698.

[14] Duda R. O. and P. E. Hart, "Use of the Hough transformation to detect lines and curves in picture", Communication. ACM, (1972), pp. 11-15.

[15] V. Mahalakshmi and S. Karthikeyan, "Clustering Based Optic Disc and Optic Cup Segmentation for Glaucoma Detection", International Journal of Innovative Research in Computer and Communication Engineering, ISSN (Print), pp. 2320-9798.

[16] http://www.ti.com/product/tms320c6416. 
International Journal of Multimedia and Ubiquitous Engineering Vol.10, No.9 (2015) 